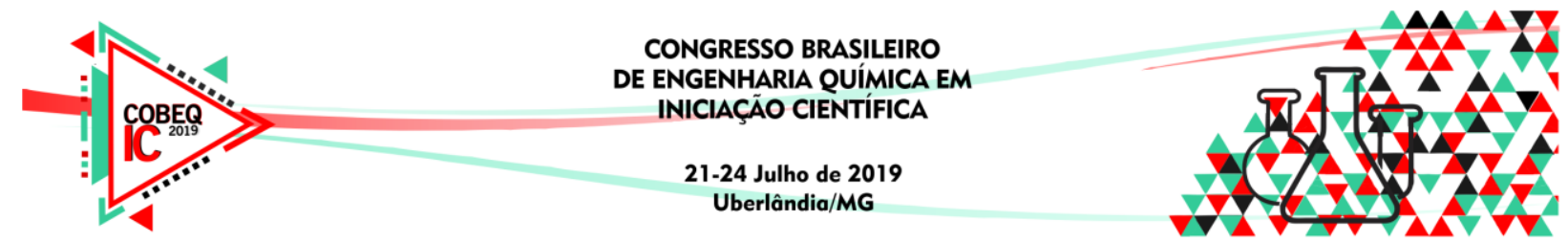

\title{
APLICAÇÃO DA LIPASE DE Rhizopus oryzae (ROL 36) IMOBILIZADA NA REAÇÃO DE ACIDÓLISE DO ÓLEO DE UVA
}

\author{
G. C. LEITE $^{1}$, O. DOMINGUES ${ }^{1}$, S. F. DIAS ${ }^{2}$, M. O. CERRI ${ }^{1}$, A. V. PAULA ${ }^{1}$
}

${ }^{1}$ Universidade Estadual Paulista, Faculdade de Ciências Farmacêuticas, Departamento de Bioprocessos e Biotecnologia

${ }^{2}$ Universidade de Lisboa, Instituto Superior de Agronomia E-mail para contato: grazycopola@ hotmail.com

\begin{abstract}
RESUMO - O processo de imobilização de enzimas é muito importante para aplicações em reações de larga escala, tendo em vista a necessidade de reutilização do biocatalisador e maior estabilidade no processo. Logo o objetivo deste trabalho foi comparar a o desempenho das lipases Rhizopus oryzae comercial e Rhizopus oryzae ROL 36 (geneticamente modificada), na reação de acidólise. O suporte utilizado para imobilização por adsorção física foi o Caulim. Foram avaliadas as atividades hidrolíticas de ambos biocatalisadores, além do desempenho de cada um deles na reação de acidificação do óleo de semente de uva e ácido cáprico (C10). Quanto à atividade hidrolítica, a lipase Rhizopus oryzae imobilizado em Caulim acidificado obteve uma melhor atividade $(1409,04$ U/g) comparado à Rhizopus oryzae ROL (1007,6 U/g). Quanto à reação de acidólise entre óleo de semente de uva e ácido cáprico, a lipase Rhizopus oryzae ROL apresentou melhores resultados de grau de incorporação (40\% e 39\%,), frente à lipase Rhizopus oryzae comercial, que foi de apenas $26,39 \%$ e $28,76 \%$.
\end{abstract}

\section{INTRODUÇÃO}

As lipases (triacilglicerol acilhidrolases, E.C. 3.1.1.3) são enzimas que apresentam diversas aplicações biotecnológicas e industriais, tais como a farmacêutica, alimentícia, têxtil, cosmética e de papel (Karl-Erich et al, 2002). Catalisam reações de hidrólise e síntese de triglicerídeos, podem ser utilizadas em diversos processos visando à obtenção de produtos oriundos de reações com lipídeos (Kappor, 2012). Esse grupo de enzimas é considerado um dos mais importantes biocatalisadores no ramo biotecnológico pela sua versatilidade de reações catalisadas (Kappor, 2012).

Em processos de larga escala, o uso de lipases imobilizadas pode ser mais vantajoso devido à estabilidade operacional, possibilidade de reuso em processos contínuos, retenção da atividade biológica por mais tempo, facilidade de separação do catalisador e do produto, redução do volume de reação, maior estabilidade ao $\mathrm{pH}$ e a temperatura (Castro et al, 2008).

Os sistemas imobilizados são influenciados tanto pelas enzimas quanto pelo material do suporte utilizado, sendo que cabe ao suporte a maior contribuição para o desempenho do sistema. Logo a escolha de um suporte deve levar em consideração as suas características 


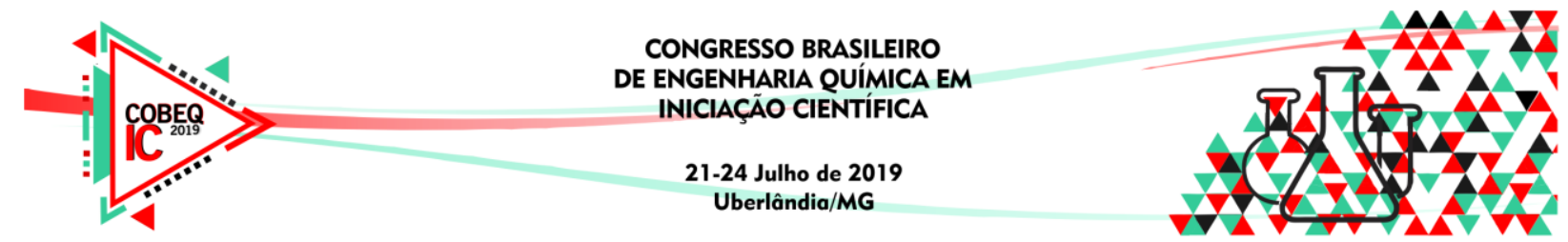

morfológicas e químicas, bem como a estabilidade química, física e possibilidade de regeneração (Castro et al, 2008). O suporte escolhido para a imobilização das lipases de Rhizopus oryzae foi o Caulim, uma argila branca, bastante empregada na indústria de papel devido à sua resistência e alvura e que, justamente por ser natural, tem grande potencial como suporte por atender a demandas ecológicas (Rahman, 2005). Neste contexto, o objetivo deste trabalho foi comparar a imobilização de diferentes lipases de Rhizopus oryzae (comercial e geneticamente modificada - ROL 36), no suporte Caulin e posteriormente empregar os derivados imobilizados na acidólise do óleo de semente de uva e ácido cáprico.

\section{MATERIAIS}

Os experimentos foram efetuados empregando preparação de lipase de Rhizopus oryzae ROL 36, geneticamente modificada, gentilmente cedida pela Professora Suzana Ferreira-Dias (Universidade de Lisboa), e lipase de Rhizopus oryzae comercial, gentilmente cedida pela Prozyn (São Paulo). O suporte utilizado foi o Caulim.

\section{METODOLOGIAS}

\subsection{Acidificação}

A reação de acidificação do suporte foi executada utilizando uma solução de ácido nítrico $1 \%$, durante 1 hora à $75^{\circ} \mathrm{C}$, Miranda (2004).

\subsection{Imobilização}

O suporte foi embebido em hexano, em proporção 1:10 (m/v), e mantido sob agitação, em temperatura ambiente, por 2 horas. Após, foram adicionados ao suporte $200 \mu \mathrm{L}$ de solução de PEG $1500(0,5 \mathrm{mg} / \mathrm{mL})$ e $0,250 \mathrm{~g}$ de enzima para cada $1 \mathrm{~g}$ de suporte. A lipase e o suporte foram mantidos em geladeira por 24 horas e posteriormente foram filtrados à vácuo e lavados com hexano (Paula, 2012).

\subsection{Determinação da Atividade Enzimática de Hidrolítica}

A determinação da atividade enzimática hidrolítica foi feita pelo método de retrotitulação, empregando-se emulsão de azeite de oliva, tal como descrito por Paula (2012).

\subsection{Reação de Acidólise}

As reações de acidólise foram realizadas entre o óleo de semente de uva e ácido cáprico, em reator de tanque agitado, catalisadas pelos dois derivados imobilizados (lipase Rhizopus oryzae imobilizada em Caulim acidificado e a lipase Rhizopus oryzae ROL 36 imobilizada em Caulim acidificado). As reações tiveram suas condições operacionais fixas (temperatura de $45^{\circ} \mathrm{C}$, razão molar óleo:ácido $1: 3$ e porcentagem de enzima de $8 \%$ em relação à massa total de meio). Foram empregados $30 \mathrm{~g}$ de meio reacional, e consequentemente $2,4 \mathrm{~g}$ de biocatalisador (lipase imobilizada, suporte + lipase) em cada reação. Os autores optaram por fixar a porcentagem de biocatalisador independentemente da atividade hidrolítica de cada 


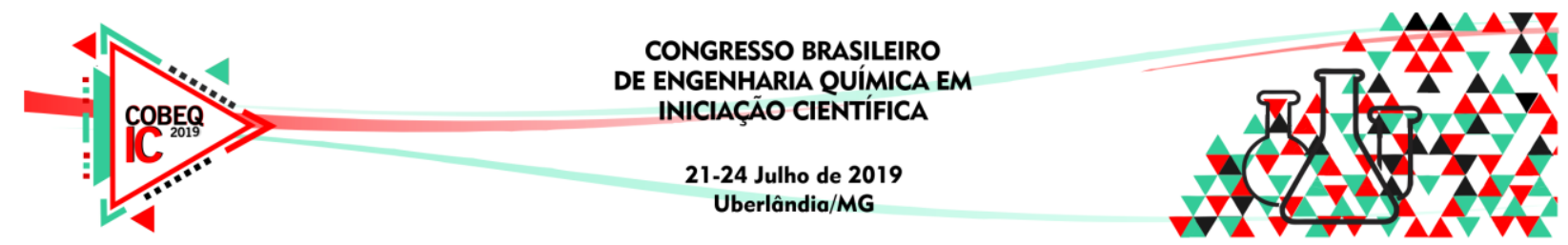

um. As reações foram conduzidas em meio isento de solventes, em reatores cilíndricos de vidro (parede dupla), com uma capacidade de $50 \mathrm{~cm}^{3}$, sob agitação magnética a $400 \mathrm{rpm}$.

\subsection{Análise do perfil em ácidos graxos}

A composição em ácidos graxos foi determinada por cromatografia gasosa, de acordo com uma modificação do método descrito por Pinese et al (2013).

\subsection{Grau de incorporação}

O grau de incorporação (GI) foi calculado de acordo com a equação 1 (CASASGODOY et al, 2012), na qual MFA é o número de mols de ácidos graxos de cadeia média (C10:0) no triglicerídeo e MT é o número de mols totais de ácidos graxos no triglicerídeo.

$$
G I(\%)=\frac{M F A}{M T} * 100
$$

\section{RESULTADOS E DISCUSSÕES}

\subsection{Atividade Hidrolítica}

As lipases Rhizopus oryzae e Rhizopus oryzae ROL 36 passaram por processos de imobilização no Caulim e realizou-se a determinação da atividade hidrolítica de cada derivado imobilizado, bem como das enzimas livres. De acordo com o item 3.2, para cada grama de suporte acidificado foram fornecidos $0,25 \mathrm{~g}$ de enzima na forma livre, para o processo de imobilização. No entanto, a metodologia de imobilização adotada faz uso de solvente orgânico (hexano), o que impossibilita a quantificação do teor de proteínas. Os dados referentes à atividade hidrolítica estão apresentados na tabela 1 .

Tabela 1- Atividade hidrolítica das lipases livres e imobilizadas em Caulim

\begin{tabular}{|c|c|c|}
\hline Enzima & $\begin{array}{c}\text { Lipase Rhizopus } \\
\text { oryzae ROL 36 }\end{array}$ & $\begin{array}{c}\text { Lipase Rhizopus } \\
\text { oryzae comercial }\end{array}$ \\
\hline \hline $\begin{array}{c}\text { Atividade Hidrolítica média } \\
\text { da enzima livre } \pm \text { Desvio } \\
\text { padrão (U/g)* }\end{array}$ & $17354,58 \pm 834,97$ & $36754,49 \pm 1591,18$ \\
\hline $\begin{array}{c}\text { Atividade Hidrolítica média } \\
\text { da enzima imobilizada } \pm \\
\text { Desvio padrão (U/g)** }\end{array}$ & $1007,60 \pm 17,54$ & $1409,04 \pm 51,07$ \\
\hline
\end{tabular}

* U/g refere-se ás unidades de atividade por grama de enzima na forma livre (pó).

** U/g refere-se às unidades de atividade por grama de derivado imobilizado (lipase imobilizada, suporte + lipase). 


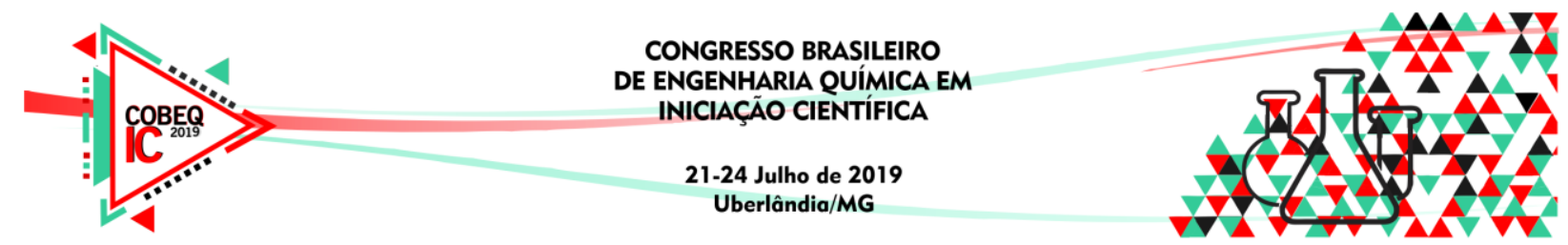

De acordo com a Tabela 1, observa-se que ao comparar a atividade hidrolítica das enzimas livres a lipase Rhizopus oryzae comercial forneceu o melhor resultado $(36754,49$ $\pm 1591,18 \mathrm{U} / \mathrm{g})$ em comparação à lipase Rhizopus oryzae ROL $36(17354,58 \pm 834,97 \mathrm{U} / \mathrm{g})$. Isso pode estar relacionado ao teor de proteínas de ambas as formulações. Esse dado não foi quantificado uma vez que o processo de imobilização posterior foi efetuado em meio orgânico (hexano). Verificando a atividade hidrolítica das enzimas imobilizadas constatou-se que a lipase Rhizopus oryzae comercial forneceu maior atividade hidrolítica $(1409,04 \pm 51,07 \mathrm{U} / \mathrm{g})$ em relação à lipase Rhizopus oryzae ROL $36(1007,6 \pm 17,54 \mathrm{U} / \mathrm{g})$. Este resultado já era esperado, uma vez que para cada grama de suporte, foram fornecidos 0,25 gramas de enzima livre, o que representa 4338,65 U e 9118,62 U de lipase Rhizopus oryzae ROL 36 e Rhizopus oryzae comercial, respectivamente.

Segundo os dados apresentados, pode-se compreender que a lipase Rhizopus oryzae comercial permitiu a obtenção dos melhores resultados de atividade hidrolítica. Relacionando os dados da enzima livre $(36754,49 \pm 1591,18 \mathrm{U} / \mathrm{g})$ com a imobilizada $(1409,04 \pm 51,07 \mathrm{U} / \mathrm{g})$ pode-se observar um decréscimo na atividade. Isso ocorre devido ao processo de imobilização que possui diversos benefícios como: estabilidade térmica e o reaproveitamento da enzima nos processos; porém, normalmente o poder de catálise da enzima do biocatalisador é diminuído (COELHO, 2008).

\subsection{Reação de Acidólise}

Realizou-se a análise do produto obtido ao final da reação de acidólise a fim de se obter o perfil de ácidos graxos em cada reação e grau de incorporação do ácido cáprico no produto. Assim, o perfil em ácidos graxos do produto reacional obtido com a Lipase Rhizopus oryzae comercial imobilizada em Caulim acidificado no reator $1(\mathrm{C} 1)$ e no reator $2(\mathrm{C} 2)$ é apresentado na Tabela 2.

Tabela 2 - Perfil em ácidos graxos presentes na amostra de óleo obtida empregando-se a lipase Rhizopus oryzae comercial imobilizada em Caulim acidificado, reator 1 (C1) e reator 2

(C2), em comparação ao óleo de semente de uva puro

\begin{tabular}{|c|c|c|c|}
\hline Reator da amostra & Ácido Graxo & $\begin{array}{c}\text { Perfil em Ácidos Graxos após } \\
\text { acidólise (\%) }\end{array}$ & $\begin{array}{c}\text { Perfil em Ácidos Graxos do Óleo de } \\
\text { semente de uva puro (\%) }\end{array}$ \\
\hline \multirow{5}{*}{ C1 } & C10 & 18,14 & - \\
& C16 & 7,39 & 13,73 \\
& C18 & 2,84 & 3,94 \\
& C18:1 & 20,68 & 24,26 \\
& C18:2 & 48,87 & 52,05 \\
& C18:3 & 2,09 & 6,02 \\
\hline \hline \multirow{5}{*}{ C2 } & C10 & 19,98 & - \\
& C16 & 7,18 & 13,73 \\
& C18 & 2,66 & 3,94 \\
& C18:1 & 19,53 & 24,26 \\
& C18:2 & 46,04 & 52,05 \\
& C18:3 & 4,62 & 6,02 \\
\hline
\end{tabular}




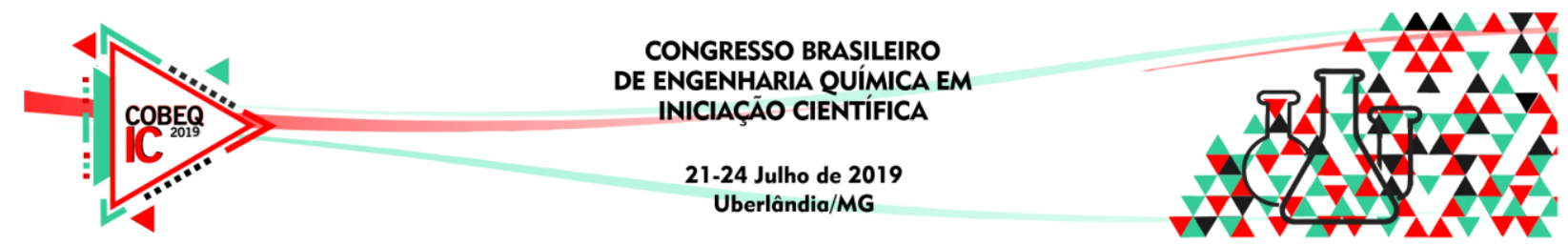

O mesmo procedimento foi realizado para o produto obtido à partir da reação empregando a da Lipase Rhizopus oryzae ROL 36 imobilizada em Caulim acidificado nos reatores $1(\mathrm{C} 3)$ e $2(\mathrm{C} 4)$. O perfil cromatográfico correspondente a eles está presente na Tabela 3.

Tabela 3 - Perfil em ácidos graxos presentes na amostra de óleo obtida empregando-se a lipase Rhizopus oryzae ROL 36 imobilizada em Caulim acidificado, reator 1 (C3) e reator 2

(C4), em comparação ao óleo de semente de uva puro

\begin{tabular}{|c|c|c|c|}
\hline Reator da amostra & Ácido Graxo & $\begin{array}{c}\text { Perfil em Ácidos Graxos após } \\
\text { acidólise }(\%)\end{array}$ & $\begin{array}{c}\text { Perfil em Ácidos Graxos do } \\
\text { Óleo de semente de uva puro } \\
(\%)\end{array}$ \\
\hline \multirow{5}{*}{ C3 } & C10 & 28,14 & - \\
& C16 & 3,2 & 13,73 \\
& C18 & 21,76 & 3,94 \\
& C18:1 & - & 24,26 \\
& C18:2 & 41,32 & 52,05 \\
& C18:3 & 3,64 & 6,02 \\
\hline \hline \multirow{5}{*}{ C4 } & C10 & 27,24 & - \\
& C16 & 3,6 & 13,73 \\
& C18 & 22,13 & 3,94 \\
& C18:1 & - & 24,26 \\
& C18:2 & 40,95 & 52,05 \\
& C18:3 & 3,59 & 6,02 \\
\hline
\end{tabular}

A partir das áreas correspondentes a cada ácido graxo, foram obtidos os valores de concentração molar, calculando-se o grau de incorporação para cada reação. Com a lipase Rhizopus oryzae comercial os valores obtidos de GI foram $26,39 \%$ e $28,76 \%$, respectivamente para C1 e C2. Já a lipase Rhizopus oryzae ROL 36 os valores obtidos de GI foram $40 \%$ e $39 \%$, respectivamente para $\mathrm{C} 3$ e $\mathrm{C} 4$.

Pode-se observar que a lipase Rhizopus oryzae ROL 36 forneceu maior grau de incorporação comparado aos valores obtidos pela lipase Rhizopus oryzae comercial. Ao se observar a composição do óleo de semente de uva puro é possível afirmar que este possui majoritariamente em sua composição ácidos graxos C18:1 (24,26\%) e C18:2 (52,05\%) e não possui o C10. Após a reação de acidólise observa-se o surgimento do ácido graxo C10 e a diminuição dos outros ácidos graxos presentes na amostra.

Além disso, deve-se considerar que as enzimas empregadas são 1,3-específica. Logo o maior GI que pode ser obtido é de 66,67\%. Portanto, os valores apresentados pela lipase comercial apresentam-se satisfatórios.

\section{CONCLUSÃO}

A imobilização das lipases de Rhizopus oryzae comercial e geneticamente modificada no suporte Caulim acidificado exposta nesse trabalho mostrou-se bem-sucedida de acordo com os resultados apresentados de atividade hidrolítica e grau de incorporação do C10 no óleo de semente de uva. 


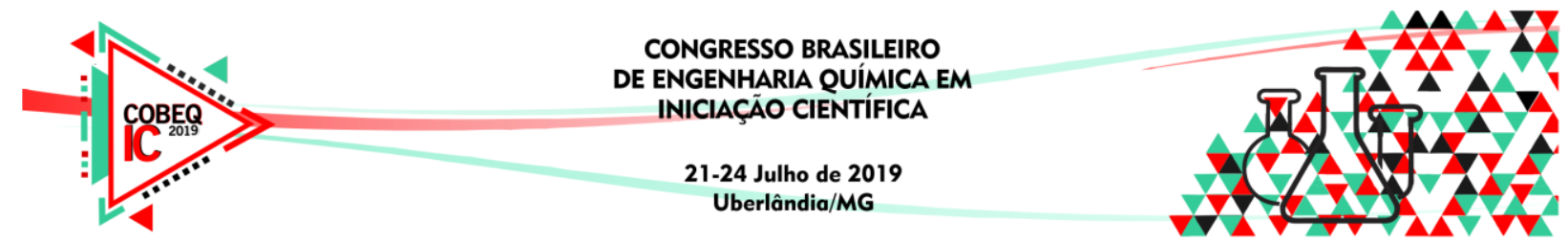

\section{REFERÊNCIAS}

CASTRO, H. F.; ZANIN, G.M.; MORAES, F. F.; SÁ-PEREIRA, P. Imobilização de enzimas e sua estabilização; In: BON, E.P.S.; FERRAR, M. A.; CORVO, M. L; Enzimas em Biotecnologia: produção, aplicações e mercado. Rio de Janeiro: Interciência, p. 123$151,2008$.

COElHO, M. A. Z., SAlGADO, A. M., RIBEIRO, B. D. Tecnologia Enzimática. EPUB. Rio de Janeiro. 2008

KAPOOR, M., GUPTA, M. N. Lipase promiscuity ands it's biochemical applications. Process Biotechnology. v.47, p. 555-569, 2012

KARL-ERICH, J., EGGERT, T., Lipases for Biotechnology. Current Opinion in Biotechnology. v.13, p. 390-397, 2002.

MIRANDA, M. Aprimoramento do processo de imobilização de lipase microbiana em óxido de nióbio para modificação de óleos vegetais. Dissertação de mestrado em Eng. Quím., Universidade de São Paulo, Lorena, 2004.

PAULA, A. V.; Reestruturação da gordura de leite por interesterificação enzimática empregando lipase imobilizada: otimização das condições reacionais e operacionais. Tese de doutorado do programa de pós-graduação de Biotec. Industrial na área de Microbiologia Aplicada, Universidade de São Paulo, Lorena, 2012.

PINESE, M., SEQUINEL, R., OLIVEIRA, J. E., Adaptação da EM 14103 para determinação da composição em ácidos graxos de óleos/gorduras e quantificação de teores de ésteres alquilicos em biodiesel numa mesma corrida cromatografica. $6^{\circ}$ Simpósio Naciona de Biocombustíveis, Canoras, Rio grande do Sul, 2013.

RAHMAN, M. B. A., TAJUDIN, S. M., HUSSEIN, M. Z., RAHMAN, R. N. Z. A., SALLEH, A. B., BASRI, M. Application of natural kaolin as support for the immobilization of lipase from Candida rugosa as biocatalyst for effective esterification. Appl. Clay Sci., v. 29, p. 111-116, 2005.

\section{AGRADECIMENTOS}

Os autores agradecem à Fundação de Amparo à Pesquisa do Estado de São Paulo (FAPESP, Processo 2017/11482-7) e à Prozyn Biosolutions for life (São Paulo, Brasil). 